\title{
An implantable cardioverter-defibrillator for primary prevention in non-ischemic cardiomyopathy: A systematic review and meta-analysis
}

\author{
Michał Wasiak $^{1,2}$, Mateusz Tajstra ${ }^{2}$, Dariusz Kosior ${ }^{3}$, Mariusz Gąsior ${ }^{2}$ \\ ${ }^{1}$ Faculty of Medical Science, Cardinal Wyszynski University in Warsaw, Poland \\ ${ }^{2} 3^{\text {rd }}$ Department of Cardiology, Silesian Center for Heart Diseases, \\ Medical University of Silesia, Zabrze, Poland \\ ${ }^{3}$ Department of Applied Physiology, Mossakowski Medical Research Center, \\ Polish Academy of Sciences, Warsaw, Poland
}

\begin{abstract}
Background: Recent data regarding the comparison of implantable cardioverter-defibrillator (ICD) therapy and optimal medical treatment in patients with non-ischemic cardiomyopathy has indicated no mortality benefit as a result of ICD therapy. Although the recommendations for ICD implantation did not change, it is worth noting that these findings significantly affected the daily practice of ICD implantation in Europe.

Methods: To assess the effect of ICD implantation in comparison to pharmacotherapy in the non-ischemic cardiomyopathy heart failure population through a systematic review and meta-analysis of the available carefully designed prospective randomized controlled trials. Only prospective randomized controlled trials comparing ICD implantation in primary prevention vs. optimal pharmacological therapy or placebo and reporting mortality results were included in the meta-analysis. The authors have chosen to include the following trials: CAT, AMIOVIRT, DEFINITE, and DANISH.

Results: A meta-analysis of pooled hazard ratios (HR) from all trials conducted on a total of 1789 patients found that ICD therapy decreased all-cause mortality in comparison to optimal pharmacological treatment, with a HR of 0.48 (95\% confidence interval [CI] 0.67-1.01); $p=0.06$. The data from the AMIOVIRT, DANISH, and DEFINITE trials, with a total of 1677 participants, showed a significant reduction of sudden cardiac deaths as a result of ICD implantation, with a HR of $0.48(95 \%$ CI $0.31-0.67) ; p<0.001$.

Conclusions: In comparison with optimal medical treatment, ICD implantation in patients with heart failure improves the long-term prognosis in terms of sudden cardiac death, with a strong tendency towards all-cause mortality reduction. (Cardiol J 2023; 30, 1: 117-124)
\end{abstract}

Key words: implantable cardioverter-defibrillator, meta-analysis, non-ischemic cardiomyopathy, systematic review

\section{Introduction}

The second half of the 1990s marked the beginning of the era of implantable devices for patients with heart failure (HF). The first credible report came from the Multicenter Automatic Defibrillator Implantation Trial (MADIT), published in 1996. The authors found a significant reduction

Address for correspondence: Michał Wasiak, MD, Department of Cardiology, Central Clinical Hospital of the Ministry of Interior and Administration, ul. Woloska 137, 02-507 Warszawa, Poland, tel: +48 2250816 70, fax: +48 225081680 , e-mail: wasiakmicha1999@gmail.com

Received: 6.07.2020 Accepted: 28.02.2021 Early publication date: 9.04.2021

This article is available in open access under Creative Common Attribution-Non-Commercial-No Derivatives 4.0 International (CC BY-NC-ND 4.0) license, allowing to download articles and share them with others as long as they credit the authors and the publisher, but without permission to change them in any way or use them commercially. 
in overall mortality associated with the implantation of a defibrillator in high-risk patients with coronary disease and left ventricular dysfunction, asymptomatic unstained ventricular tachycardia, and inducible sustained ventricular tachycardia. Patients randomly assigned to receive the defibrillator had a much lower rate of death from primary arrhythmia than patients assigned to conventional therapy [1]. Since then, large randomized clinical trials have established the role of implantable cardioverter-defibrillators (ICDs) in primary prevention, which was reflected in the updated European and American guidelines that assign a class I recommendation for prophylactic ICD therapy in patients with $\mathrm{HF}$ and reduced ejection fraction, regardless of HF etiology [2-5].

Published in 2016, the report from the Defibrillator Implantation in Patients with Non-ischemic Systolic Heart Failure (DANISH) trial questions the benefits an ICD implantation in patients with non-ischemic HF etiology (NICM). A comparison of ICD therapy with optimal medical treatment (OMT) in patients with NICM reported no mortality benefit as a result of ICD therapy [6]. Although the recommendations for ICD implantation did not change after the publication of the DANISH trial results, it is worth noting that these findings significantly affected the daily practice of ICD implantation in Europe. According to the European Heart Rhythm Association survey, many European medical centers introduced changes in their indications for ICD therapy after the publication of the DANISH trial results. The DANISH study succeeded in changing physicians' attitudes regarding ICD indications in the NICM population [7].

In light of the above, along with recently published findings, we aimed to assess the effect of ICDs implanted in the primary prevention of sudden cardiac death (SCD), in comparison to pharmacotherapy, in the NICM heart failure population through a systematic review of the results of available credible randomized controlled trials (RCTs) and a meta-analysis.

\section{Methods}

Our systematic review and meta-analysis were based on established methods recommended by the Cochrane guidelines, and they remain in compliance with the Preferred Reporting Items for Systematic Reviews and Meta-analyses (PRISMA) statement [8].

\section{Data sources and searches}

The authors searched sources published between February1980 and January 2020, including MEDLINE, EMBASE, PUBMED, the Cochrane Central Register of Controlled Trials, and www. clinicaltrials.gov, without language restriction. In February 1980, Dr. Levi Watkins implanted the first ICD at Johns Hopkins Hospital. All reports regarding RCT and their evaluations were published after this date. When searching the clinical trials registers, we used the following keywords: nonischemic cardiomyopathy, implantable cardioverter defibrillator, dilated cardiomyopathy.

\section{Study selection}

Only prospective RCTs including a comparison of ICD implantation in primary prevention vs. optimal pharmacological therapy or placebo and reporting mortality results were included in the meta-analysis. Non-randomized, single group studies were excluded. Previously published metaanalyses and systematic reviews that correspond with our study selection were included in our review. The PRISMA flow chart showing the publication screening process is depicted in Figure 1.

\section{Data collection, extraction, analyses, and quality assessment}

We undertook a meta-analysis of the studies where it was reasonable to do so, namely if the protocol, participants, and the clinical question were comparable enough for pooling data.

We extracted the following data and characteristics:

- methods: study design, follow-up duration;

- participants: inclusion and exclusion criteria, number of individuals, clinical characteristics (e.g. age, gender, left ventricular ejection fraction [LVEF], New York Heart Association [NYHA] classification, comorbidities);

- medical interventions: ICD implantation, optimal pharmacological therapy, placebo, concomitant medications;

- outcomes: primary and secondary outcomes. Hazard ratios (HRs) with 95\% confidence intervals (CIs) were abstracted for all available mortality outcomes. We pooled the log HRs for time-to-event data and used the generic inverse variance method for statistical computations and graphics in Review Manager 5 software. A p value of 0.05 was set as significant. The inconsistency index $\left(\mathrm{I}^{2}\right)$ and $\mathrm{p}$ values were used to quantify het- 


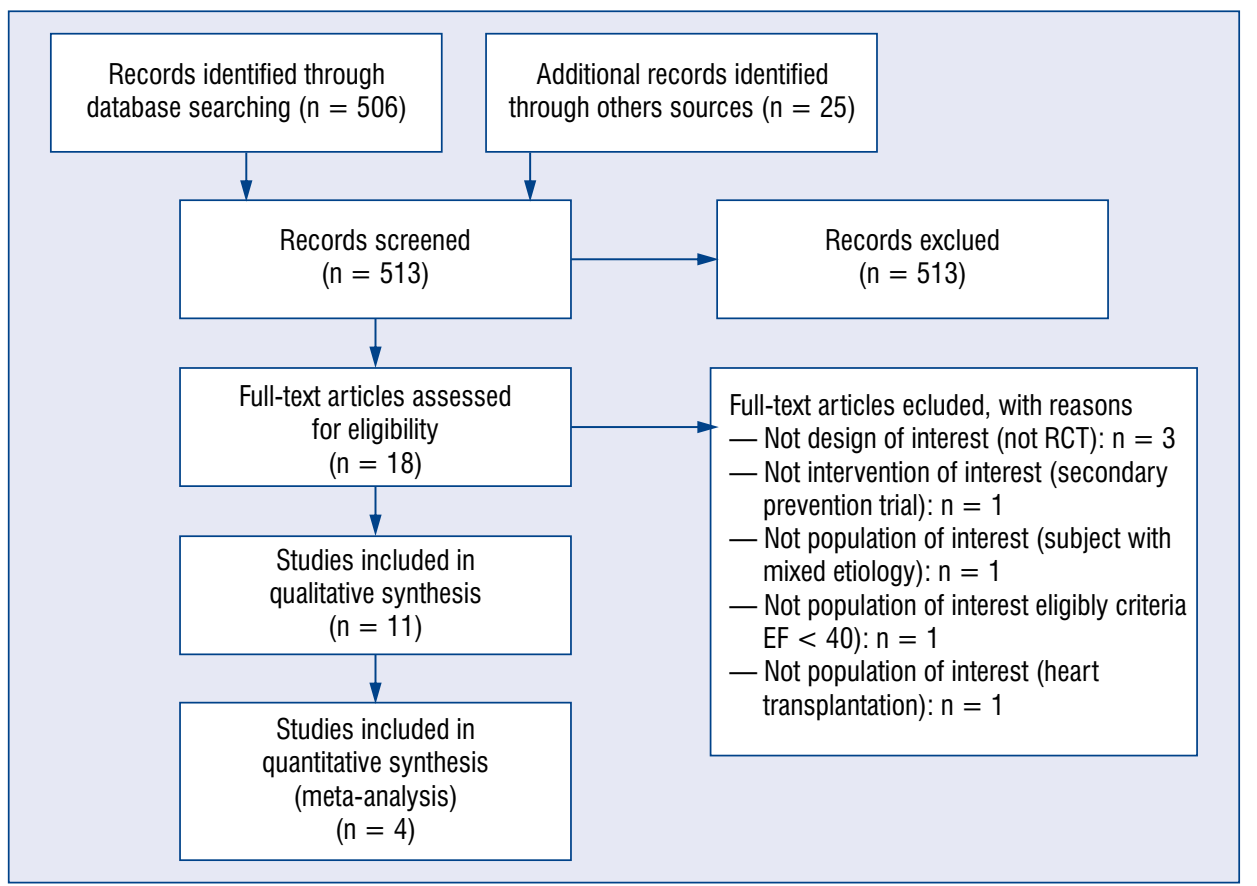

Figure 1. Study flow diagram; EF — ejection fraction; RCT — randomized controlled trials.

erogeneity. In our study a fixed effect was used during the statistical analyses.

The risk of bias assessment for each study was performed according to the latest revised Cochrane risk-of-bias tool for randomized trials (RoB 2). All types of bias that could affect the results of randomized trials (bias arising from the randomization process due to deviations from intended interventions or missing outcome data, bias in the measurement of the outcome or in the selection of the reported result) were considered. We graded each potential source of bias as low, high, or unclear, and provided a quote from the study report, along with a justification for our judgement, in the 'Risk of bias' table (Table 1 ).

\section{The role of the funding source}

None of the investigators involved in the review received any funding. No external organizations or individuals were involved in any aspect of the research.

\section{Results}

The authors decided to include the results of the following trials in the meta-analysis: Cardiomyopathy Trial (CAT), Amiodarone Versus Implantable Cardioverter-Defibrillator trial (AMIOVIRT), Defibrillators in Non-Ischemic Cardiomyopathy Treat- ment Evaluation Trial (DEFINITE), and DANISH [6, 9-11]. They are the only identified RCTs in which the experimental and control group were NICM. The baseline clinical characteristics of the study group are presented in Table 2 .

The 4 trials recruited a total of 1789 participants. All studies included adult patients with the mean age of the study population ranging from 52 (CAT) to 64 years (DANISH). The follow-up period in 3 studies was similar (from 22 to 26 months), while the DANISH trial reported a substantially longer follow-up period, namely 56 months. All studies reported sex disproportion, with $70-80 \%$ of study participants being men. The mean LVEF was comparable through all trials, varying from $21.4 \%$ in DEFINITE to $25 \%$ in DANISH. AMIOVIRT and DEFINITE included patients in class I-III NYHA, CAT - in class III-IV, and DANISH - in class II-IV. Regarding comorbidities, the CAT trial presented only the atrial fibrillation burden $(15.7 \%)$. However, in this trial alone, the entire study population underwent an electrophysiological examination. Inducible ventricular tachycardia was present in $2.9 \%$ of the cases and ventricular fibrillation - in $9.6 \%$. Pharmacotherapy was substantially diverse across the reports. Beta-blocker administration ranged from 3.8\% (CAT) to $92 \%$ (DANISH). Aldosterone antagonist pharmacotherapy varied from $19 \%$ to $59 \%$ (AMIOVIRT 


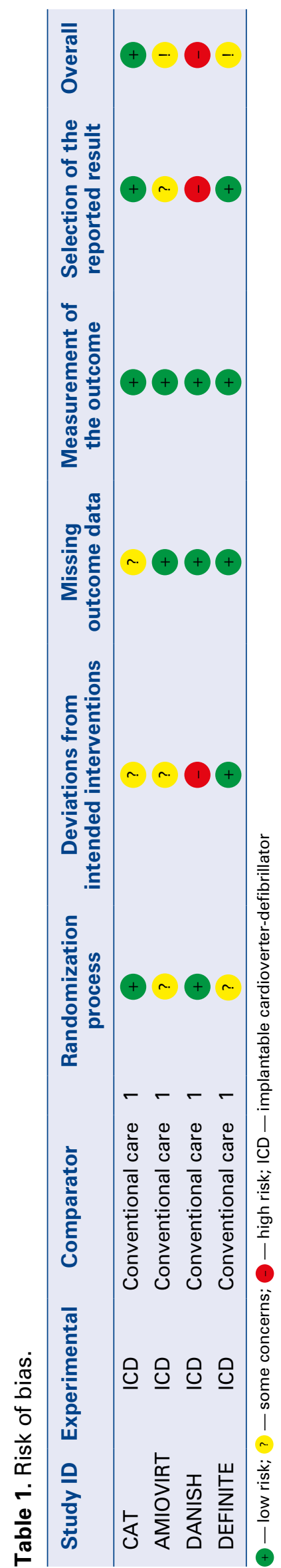

and DANISH, respectively), while in the CAT and DEFINITE trials it was not reported at all. Angiotensin-converting-enzyme inhibitors were widely used in all trials and rated $>80 \%$.

\section{Primary endpoint}

The meta-analysis of pooled hazard ratios in all trials, including a total of 1789 patients, showed that all-cause mortality in the ICD group decreased with borderline significance (167 of 886 patients, i.e. $19 \%$ ) in comparison to optimal pharmacological treatment (195 of 895 patients, i.e. $22 \%$ ), with a HR of 0.48 (95\% CI 0.67-0.10); $\mathrm{p}=0.06$; heterogeneity $\mathrm{p}=0.78 ; \mathrm{I}^{2}=0 \%$ (Fig. 2).

\section{Secondary endpoint}

Sudden cardiac deaths were not reported in the CAT trial. The data from AMIOVIRT, DANISH, and DEFINITE, including a total of 1678 participants, showed a significant reduction of SCDs owing to ICD implantation: 28 of 436 patients vs. 62 of 841 patients (ICD and conventional care, respectively); with a HR of 0.48 (95\% CI 0.31-0.67); $\mathrm{p}<0.001$; heterogeneity $\mathrm{p}=0.39 ; \mathrm{I}^{2}=0 \%$ (Fig. 3 )

\section{Health-related quality of life}

A total of 561 participants in the analyzed RCTs underwent the quality-of-life evaluation (AMIOVIRT and DEFINITE). Investigators used the following scales: the Quality of Well-Being Schedule $(n=1)$, the Minnesota Living With Heart Failure Questionnaire (MLHFQ), the 12-Item Short Form Health Survey (SF-12), and the State-Trait Anxiety Inventory. No significant differences in the quality of life between two groups were noted, which suggests that ICD therapy has no measurable effect on a patient's quality of life.

“Quality assessment". All of the 4 studies included in this meta-analysis had low risk of bias regards measurement of the outcome. High risk of bias was noticed in deviations from intended interventions and selection of the reported result in the DANISH trial. Five percent of patients in the control group received ICD, and $8 \%$ of patients in the ICD group did not undergo implantation, or had the device deactivated or extracted. The selection of the reported result also raised concerns. The DANISH trial approved the implantation of a CRT device in all eligible patients and then randomized the study population to the CRT-D or CRT-P arm. Results from patients who required resynchronization therapy were extrapolated to SCD primary prevention indications. 
Table 2. Baseline clinical characteristics.

\begin{tabular}{|c|c|c|c|c|}
\hline & \multicolumn{4}{|c|}{ Trial name and year of publication } \\
\hline & CAT 2002 & AMIOVIRT 2003 & DEFINITE 2004 & DANISH 2016 \\
\hline No participants & 104 & 103 & 458 & 1116 \\
\hline Age, mean \pm SD [year] & $52 \pm 11$ & $59 \pm 11.5$ & $58 *$ & $64^{*}$ \\
\hline Follow-up, mean $\pm \mathrm{SD}$ [month] & $66 \pm 26.4$ & $24 \pm 14.4$ & $29 \pm 14$ & $67.6 \pm N R$ \\
\hline Male & $83(80 \%)$ & $72(70 \%)$ & $326(71 \%)$ & $809(72 \%)$ \\
\hline LVEF, mean \pm SD $[\%]$ & $23 \pm 9$ & $21 \pm 14$ & $24 \pm 7$ & $25 \pm N R$ \\
\hline ACEI/ARB & $100(96.2 \%)$ & $88(85 \%)$ & $443(96.7 \%)$ & $1077(97 \%)$ \\
\hline Beta-blockers & $4(3.8 \%)$ & $53(51.5 \%)$ & $389(84.9 \%)$ & $1026(92 \%)$ \\
\hline MRA & NR & $19.4(20 \%)$ & NR & $58(646 \%)$ \\
\hline Atrial fibrillation & $16(15.7 \%)$ & NR & 112 & 248 \\
\hline Arterial hypertension & NR & 64 & NR & 348 \\
\hline Diabetes mellitus & NR & 35 & 105 & 211 \\
\hline
\end{tabular}

${ }^{*}$ Calculated as mean of stated medians for treatment and control groups; ACEI — angiotensin-converting enzyme inhibitor; AMIOVIRT — Amiodarone Versus Implantable Cardioverter-Defibrillator; ARB - angiotensin-receptor blocker; CAT — Cardiomyopathy Trial; DANISH Danish Study to Assess the Efficacy of ICDs in Patients with Non-ischemic Systolic Heart Failure on Mortality; DEFINITE - Defibrillators in Non-Ischemic Cardiomyopathy Treatment Evaluation Trial; LVEF — left ventricular ejection fraction; MRA — mineralocorticoid receptor antagonist; NR - not reported

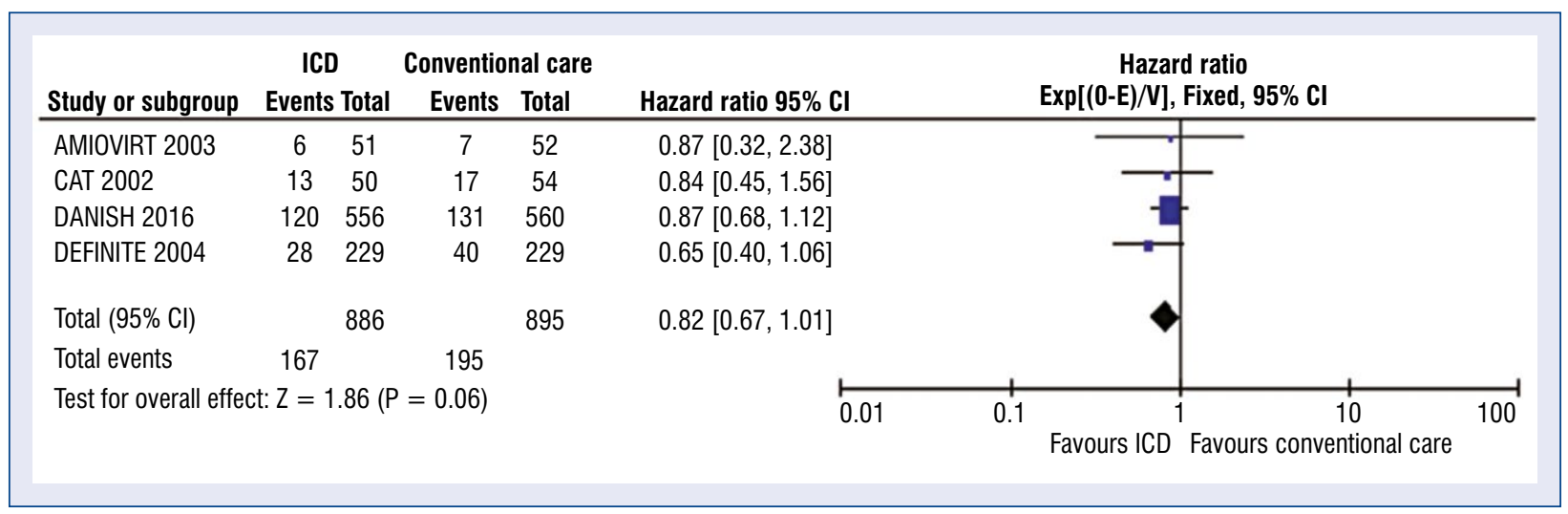

Figure 2. Hazard ratios for all-cause death: implantable cardioverter-defibrillator (ICD) therapy versus conventional care; AMIOVIRT — Amiodarone Versus Implantable Cardioverter-Defibrillator: Randomized Trial in Patients with Nonischemic Dilated Cardiomyopathy and Asymptomatic Non-sustained Ventricular Tachycardia; CAT — Cardiomyopathy Trial; $\mathrm{Cl}$ - confidence interval; DANISH - Danish Study to Assess the Efficacy of ICDs in Patients with Non-ischemic Systolic Heart Failure on Mortality; DEFINITE — Defibrillators in Non-Ischemic Cardiomyopathy Treatment Evaluation; ICD — implantable cardioverter-defibrillator.

\section{Discussion}

\section{Meta-analysis}

The main finding of our meta-analysis indicates a significant reduction in the SCD rate among ICD recipients and a strong tendency towards all-cause mortality reduction in comparison to optimal pharmacological treatment alone.

Our findings support the current and binding European and American recommendations regarding ICD implantation in the NICM population.
A few meta-analyses have been published in this field in recent years. One of the first was a publication presented by Desai et al. [12] with pooled data from 5 trials including patients with a non-ischemic and mixed etiology of HF (AMIOVIRT, CAT, DEFINITE, COMPANION [Comparison of Medical Therapy, Pacing, and Defibrillation in Heart Failure Trial], and SCD-HeFT [Sudden Cardiac Death in Heart Failure Trial]). The authors presented a statistically significant $31 \%$ reduction in all-cause mortality within the ICD population 


\begin{tabular}{|c|c|c|c|c|c|c|c|}
\hline \multirow{3}{*}{$\begin{array}{l}\text { Study or subgroup } \\
\text { AMIOVIRT } 2003\end{array}$} & \multicolumn{2}{|c|}{ ICD } & \multicolumn{2}{|c|}{ Conventional care } & \multirow[b]{2}{*}{ Hazard ratio, Fixed $95 \%$ Cl } & \multirow{2}{*}{\multicolumn{2}{|c|}{$\begin{array}{c}\text { Hazard ratio } \\
\text { Exp }[(0-\mathrm{E}) / \mathrm{V}], \text { Fixed, } 95 \% \mathrm{Cl} \\
\end{array}$}} \\
\hline & Events & Total & Events & Total & & & \\
\hline & 1 & 51 & 2 & 52 & $0.50[0.24,1.04]$ & 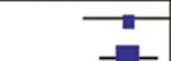 & \\
\hline DANISH 2016 & 24 & 556 & 46 & 560 & $0.50[0.31,0.81]$ & -1 & \\
\hline DEFINITE 2004 & 3 & 229 & 14 & 229 & $0.20[0.06,0.69]$ & & \\
\hline Total $(95 \% \mathrm{Cl})$ & \multirow[b]{2}{*}{28} & 836 & & 841 & \multirow[t]{2}{*}{$0.46[0.31,0.67]$} & & \\
\hline Total events & & & 62 & & & & \\
\hline \multirow{2}{*}{\multicolumn{4}{|c|}{ Test for overall effect: $Z=3.98(P<0.0001)$}} & & 0.01 & 0.1 & $10 \quad 100$ \\
\hline & & & & & & Favours ICD & Favours conventional care \\
\hline
\end{tabular}

Figure 3. Hazard ratios for sudden death: implantable cardioverter-defibrillator therapy versus conventional care; AMIOVIRT - Amiodarone Versus Implantable Cardioverter-Defibrillator: Randomized Trial in Patients with Non-ischemic Dilated Cardiomyopathy and Asymptomatic Non-sustained Ventricular Tachycardia; $\mathrm{Cl}$ - confidence interval; DANISH - Danish Study to Assess the Efficacy of ICDs in Patients with Non-ischemic Systolic Heart Failure on Mortality; DEFINITE — Defibrillators in Non-Ischemic Cardiomyopathy Treatment Evaluation; ICD — implantable cardioverter-defibrillator.

relative to OMT (risk ratio $=0.69 ; 95 \%$ CI $0.55-$ $-0.87 ; \mathrm{p}=0.002$ ) [12]. A similar study published by Romero et al. [13] on the basis of the AMIOVIRT, CAT, DEFINITE, COMPANION, SCD-HeFT, and DANISH trials reported a significant reduction in all-cause mortality in the ICD group compared with OMT (risk ratio $=0.79,95 \%$ CI $0.68-0.92$; $\mathrm{p}=0.002$ ).

Al-Khatib et al. [14] analyzed 4 randomized clinical trials (CAT, DEFINITE, SCD-HeFT, and DANISH), which included 1874 patients with NICM. Pooled data from these trials showed a significant reduction in all-cause mortality in the ICD group $(\mathrm{HR}=0.75 ; 95 \%$ CI $0.61-0.93$; $\mathrm{p}=0.008)$. The authors excluded the AMIOVIRT trial, which was not discussed in the publication.

Although the results of the trials mentioned above coincide with our findings, some differences in methodology should be noted. The authors of those reports included the results from the COMPANION and SCD-HeFT trials, although the study groups in those trials included patients with ischemic and non-ischemic heart failure etiology [12].

A detailed review was presented by El Moheb et al. [15]. In their meta-analysis of 6 randomized trials including a total of 3128 participants, the authors found that the use of ICD vs. OMT alone decreased the risk of all-cause mortality $(\mathrm{HR}=0.78$, 95\% CI 0.66-0.9). An average of 24 patients needed to be treated with ICD to prevent one additional death from any cause. Patients under the age of 65 years gained more benefit than individuals older than 65 years $(\mathrm{HR}=0.51,95 \% \mathrm{CI} 0.29-0.91)$.
Compared to these meta-analyses, our study covers all up-to-date RCTs in which the study population consisted exclusively of patients with NICM. Furthermore, our study consistently followed the latest Cochrane guidelines regarding a comprehensive risk of bias evaluation.

The results of the DANISH trial should be assessed by taking into account the fact that modes of death vary with age. SCD rates are roughly similar among younger and older patients, while the rate of non-SCDs is twice as high in the older population, as reported by Elming et al. [16]. There was a relation between reduced all-cause mortality and ICD therapy in the population of patients $\leq 70$ years of age $(\mathrm{HR}=0.70 ; 95 \% \mathrm{CI}$ $0.51-0.96 ; p=0.03)$, while the population of patients $>70$ years of age did not show this correlation $(\mathrm{HR}=1.05 ; 95 \% \mathrm{CI} 0.68-1.62 ; \mathrm{p}=0.84)$. For patients $\leq 70$ years of age, the SCD rate was 1.8 (95\% CI 1.3-2.5) and the non-sudden death rate was 2.7 (95\% CI 2.1-3.5), while for patients $>70$ years of age, the SCD rate was $1.6(95 \% \mathrm{CI}$ $0.8-3.2)$ and the non-sudden death rate was 5.4 $(95 \%$ CI 3.7-7.8, $\mathrm{p}=0.01)$.

Moreover, the substantial risk of bias in the DANISH trial results from the fact that the DANISH trial approved the implantation of a CRT device in all eligible patients and then randomized the study population to the CRT-D or CRT-P arm. Considering that $65 \%$ of older patients received a CRT device, it is likely that the ICD therapy impact on survival was diluted by the use of the CRT device in both arms, which resulted in the lack of statistical power to 
present a discrepancy in the survival of patients with ICDs, who were not eligible to receive a CRT device.

\section{Registries}

The presented meta-analysis encompasses prospective RCTs in which the study and control groups consisted entirely of patients with NICM. It is worthwhile mentioning some recently published registries that included patients with NICM and ICD implantation. Having analyzed the retrospective, propensity-matched WARCEF trial (Warfarin vs. Aspirin in Reduced Cardiac Ejection Fraction), with a mean follow-up of $3.5 \pm 1.8$ years, the authors noted that the presence of an ICD at baseline was associated with a lower risk of all-cause death among those with ischemic cardiomyopathy ( $\mathrm{HR}=$ $=0.640 ; 95 \%$ CI $0.45-0.91 ; \mathrm{p}=0.015)$, but not among those with NICM (HR $=0.984 ; 95 \%$ CI $0.64-1.51$; $\mathrm{p}=0.9$ ) [17]. The major weakness of this study was its lack of data regarding the group without ICD implantation. The authors did not report why this group (subtracted from the WARCEF population) was not initially qualified to ICD implantation. No data relating to primary and secondary prevention were provided.

The authors of this publication recently presented the findings of the COMMIT-HF (Contemporary Modalities in the Treatment of Heart Failure) registry. A comparison between ischemic and non-ischemic HF etiology among ICD recipients was conducted from January 2009 to December 2013 with a median of 60.5 months. We observed a significantly better clinical profile within the non-ischemic group, with a lower mortality rate. Furthermore, we discovered that ischemic etiology is a strong independent predictor of all-cause mortality after ICD implantation [18].

\section{Limitations of the study}

Our review and meta-analysis have several limitations. The follow-up of the included trials differs substantially, the $\mathrm{p}$ value of heterogeneity was nonsignificant, and different medical therapies were used across the trials.

\section{Conclusions}

In conclusion, based on the currently available data, ICD implantation as a primary SCD prevention measure is well proven by randomized controlled trials. Our meta-analysis strongly supports the legitimacy of ICD implantation among patients with NICM.

\section{Conflict of interest: None declared}

\section{References}

1. Moss AJ, Hall WJ, Cannom DS, et al. Improved survival with an implanted defibrillator in patients with coronary disease at high risk for ventricular arrhythmia. Multicenter Automatic Defibrillator Implantation Trial Investigators. N Engl J Med. 1996; 335(26): 1933-1940, doi: 10.1056/NEJM199612263352601, indexed in Pubmed: 8960472.

2. Moss AJ, Zareba W, Hall WJ, et al. Prophylactic implantation of a defibrillator in patients with myocardial infarction and reduced ejection fraction. N Engl J Med. 2002; 346(12): 877-883, doi: 10.1056/NEJMoa013474, indexed in Pubmed: 11907286.

3. Bardy GH, Lee KL, Mark DB, et al. Amiodarone or an implantable cardioverter-defibrillator for congestive heart failure. N Engl J Med. 2005; 352(3): 225-237, doi: 10.1056/NEJMoa043399, indexed in Pubmed: 15659722.

4. Ponikowski P, Voors AA, Anker SD, et al. 2016 ESC Guidelines for the diagnosis and treatment of acute and chronic heart failure: The Task Force for the diagnosis and treatment of acute and chronic heart failure of the European Society of Cardiology (ESC). Developed with the special contribution of the Heart Failure Association (HFA) of the ESC. Eur J Heart Fail. 2016; 18(8): 891-975, doi: 10.1002/ejhf.592, indexed in Pubmed: 27207191.

5. Yancy CW, Jessup M, Bozkurt B, et al. 2017 ACC/AHA/HFSA Focused Update of the 2013 ACCF/AHA Guideline for the Management of Heart Failure: A Report of the American College of Cardiology/American Heart Association Task Force on Clinical Practice Guidelines and the Heart Failure Society of America. Circulation. 2017; 136(6): e137-e161, doi: 10.1161/ CIR.0000000000000509, indexed in Pubmed: 28455343.

6. Køber L, Thune JJ, Nielsen JC, et al. Defibrillator Implantation in Patients with Nonischemic Systolic Heart Failure. N Engl J Med. 2016; 375(13): 1221-1230, doi: 10.1056/NEJMoa1608029, indexed in Pubmed: 27571011.

7. Haugaa KH, Tilz R, Boveda S, et al. Implantable cardioverter defibrillator use for primary prevention in ischaemic and nonischaemic heart disease-indications in the post-DANISH trial era: results of the European Heart Rhythm Association survey. Europace. 2017; 19(4): 660-664, doi: 10.1093/europace/eux089, indexed in Pubmed: 28431077.

8. Liberati A, Altman DG, Tetzlaff J, et al. The PRISMA statement for reporting systematic reviews and meta-analyses of studies that evaluate health care interventions: explanation and elaboration. PLoS Med. 2009; 6(7): e1000100, doi: 10.1371/journal. pmed.1000100, indexed in Pubmed: 19621070.

9. Bansch D, Antz M, Boczor S. Primary prevention of sudden cardiac death in idiopathic dilated cardiomyopathy. the cardiomyopathy trial (CAT). ACC Current J Rev. 2002; 11(5): 73, doi: 10.1016/s1062-1458(02)00817-6.

10. Strickberger S, Hummel J, Bartlett T, et al. Amiodarone versus implantable cardioverter-defibrillator:randomized trial in patients with nonischemicdilated cardiomyopathy and asymptomaticnonsustained ventricular tachycardia - AMIOVIRT. J Am Coll Cardiol. 2003; 41(10): 1707-1712, doi: 10.1016/s0735-1097(03)00297-3.

11. Kadish A, Dyer A, Daubert JP, et al. Prophylactic defibrillator implantation in patients with nonischemic dilated cardiomyopathy. N Engl J Med. 2004; 350(21): 2151-2158, doi: 10.1056/ NEJMoa033088, indexed in Pubmed: 15152060.

12. Desai AS, Fang JC, Maisel WH, et al. Implantable defibrillators for the prevention of mortality in patients with nonischemic cardiomyopathy. A meta-analysis of randomized controlled tri- 
Cardiology Journal 2023, Vol. 30, No. 1

als. ACC Current J Rev. 2005; 14(3): 47, doi: 10.1016/j.accreview.2005.02.022.

13. Romero J, Díaz JC, Grushko M, et al. Clinical impact of implantable cardioverter-defibrillator in primary prevention of total mortality in non-ischaemic cardiomyopathy: results from a meta-analysis of prospective randomized clinical trials. Europace. 2018; 20(FI2): f211-f216, doi: 10.1093/europace/eux324, indexed in Pubmed: 29161424.

14. Al-Khatib S, Fonarow G, Joglar J, et al. Primary prevention implantable cardioverter defibrillators in patients with nonischemic cardiomyopathy. JAMA Cardiol. 2017; 2(6): 685, doi: 10.1001/ jamacardio.2017.0630.

15. El Moheb M, Nicolas J, Khamis AM, et al. Implantable cardiac defibrillators for people with non-ischaemic cardiomyopathy. Cochrane Database Syst Rev. 2018; 12: CD012738, doi: 10.1002/14651858. CD012738.pub2, indexed in Pubmed: 30537022.
16. Elming MB, Nielsen JC, Haarbo J, et al. Age and outcomes of primary prevention implantable cardioverter-defibrillators in patients with nonischemic systolic heart failure. Circulation. 2017; 136(19): 1772-1780, doi: 10.1161/CIRCULATIONAHA.117.028829, indexed in Pubmed: 28877914.

17. Lee TC, Qian M, Mu L, et al. Association between mortality and implantable cardioverter-defibrillators by aetiology of heart failure: a propensity-matched analysis of the WARCEF trial. ESC Heart Fail. 2019; 6(2): 297-307, doi: 10.1002/ehf2.12407, indexed in Pubmed: 30816013.

18. Wasiak M, Tajstra M, Pyka $€$, et al. Long-term clinical outcomes after placement of an implantable cardioverter-defibrillator: does the etiology of heart failure matter? Kardiol Pol. 2020; 78(4): 318-324, doi: 10.33963/KP.15242, indexed in Pubmed: 32200617. 